\title{
Mise en évidence d'une similitude d'action fongicide entre le fenpiclonil, l'iprodione et le tolclofos-méthyl
}

\author{
$P$ Leroux \\ INRA, station de phytopharmacie, route de Saint-Cyr, 78026 Versailles, France
}

(Reçu le 6 décembre 1990; accepté le 19 décembre 1990)

\begin{abstract}
Résumé - Le fenpiclonil est un nouveau fongicide de la famille des phénylpyrroles. Chez Botrytis cinerea son effet fongitoxique est similaire à celui de l'iprodione au moment de la germination des spores. Par ailleurs, il existe une résistance croisée entre le fenpiclonil, l'iprodione et le tolclofos-méthyl chez certaines souches de ce parasite ainsi que chez Penicillium expansum et Pseudocercosporella herpotrichoides. Ces résultats suggèrent que les dicarboximides comme l'iprodione, des dérivés aromatiques comme le tolclofos-méthyl et des phénylpyrroles comme le fenpiclonil ont le même mode d'action biochimique chez les champignons.
\end{abstract}

dicarboximide / phénylpyrrole / résistance / Botrytis cinerea / Penicillium expansum / Pseudocercosporella herpotrichoides

Summary - Similarity between the antifungal activities of fenpiclonil, iprodione and tolclofos-methyl. Fenpiclonil is a new phenylpyrrole fungicide which in Botrytis cinerea during spore germination, has a fungitoxic effect similar to that produced by iprodione. Moreover, a positive cross-resistance is observed between fenpiclonil, iprodione and tolclofos-methyl in some strains of the $B$ cinerea and also in Penicillium expansum and Pseudocercosporella herpotrichoides. Such results suggest that dicarboximides like iprodione, aromatic derivative such as tolclofos-methyl and phenylpyrroles like fenpiclonil have a similar biochemical mode of action in fungi.

dicarboximide / phenylpyrrole / resistance / Botrytis cinerea / Penicillium expansum / Pseudocercosporella herpotrichoides

\section{INTRODUCTION}

Le fenpiclonil ou CGA 142705 [4-(2,3dichlorophényl)pyrrole-3-carbonitrile] est un fongicide récemment développé en traitements de semences des céréales pour combattre Fusarium nivale, Tilletia caries, Septoria nodorum, Pyrenophora graminea et Pyrenophora teres. Sur d'autres cultures il serait actif vis-à-vis de Rhizoctonia solani, Alternaria spp et Aschochyta spp (Nevill et al, 1988). Un autre phénylpyrrole : le CGA 173506 [4-(2,2 difluoro-1,3-benzodioxol-4yl) pyrrole-3-carbonitrile] présente des propriétés similaires au fenpiclonil en traitements de semences et il est, en outre, applicable en cours de végétation contre des champignons appartenant aux genres Botrytis, Monilinia et Sclerotinia (Gehmann et al, 1990). Ces 2 fongicides "phénylpyrroles" synthétiques sont des analogues structuraux d'un antibiotique : le pyrrolnitrin isolé de Pseudomonas pyrrocinia et utilisé comme antimycotique chez les animaux (Lyr, 1977).

Les spectres d'activité de ces fongicides phénylpyrroles étant relativement voisins de ceux de la famille des dicarboximides (iprodione, procymidone, vinchlozoline, ...) et de certains dérivés aromatiques (dicloran, quintozène, tolclofosméthyl, ...) (Leroux et Fritz, 1984), il est possible d'imaginer que tous ces fongicides ont un même mode d'action. Divers essais ont été conduits avec le fenpiclonil, l'iprodione et le tolclofosméthyl sur Botrytis cinerea, Penicillium expansum et Pseudocercosporella herpotrichoides afin de vérifier cette hypothèse.

\section{MATÉRIEL ET MÉTHODE}

Parmi les 17 souches de $B$ cinerea étudiées, 5 (BS) étaient sensibles aux dicarboximides, 10 $(\mathrm{Br})$ issues du vignoble champenois étaient moyennement résistantes aux dicarboximides et 2 (BR) correspondaient aux mutants SUG2 et SUG8 induits en laboratoire en présence d'iprodione (Leroux et al, 1978). Chez $P$ expansum les essais ont porté sur 2 souches sensibles (PS) à l'iprodione et sur 2 mutants (PR) induits en laboratoire, dont POG2 décrit par Leroux et al (1978). 
Quant aux 12 souches (types HS, $\mathrm{Hr} 1$ ou $\mathrm{Hr} 2$ ) de $P$ herpotrichoides, elles présentaient une croissance rapide et avaient été isolées en France sur blé tendre d'hiver (Leroux et Gredt, 1989).

Le fenpiclonil, l'iprodione et le tolclofos-méthyl, présentés sous forme de produits techniques, nous avaient été aimablement fournis respectivement par Ciba-Geigy Agro (Bâle, Suisse), Rhône-Poulenc Agro (Lyon, France) et Sumitomo Chemical (Osaka, Japon).

L'effet des fongicides sur la croissance mycélienne des diverses souches de champignons cultivées sur milieu gélosé a été étudié selon la méthode de Leroux et al (1978). Les diverses notations obtenues dans ces essais ont permis d'estimer les concentrations en fongicide inhibant de $50 \%\left(\mathrm{Cl}_{50}\right)$ la vitesse de croissance mycélienne. Les niveaux de résistance ont été ensuite calculés en faisant les rapports des $\mathrm{Cl}_{50}$ obtenues respectivement sur souches résistantes et sensibles. Chez $B$ cinerea, des essais ont de plus été conduits pour déterminer les concentrations inhibant de $50 \%$ la germination des spores ou l'élongation des filaments germinatifs (Leroux et Gredt, 1989).

\section{RÉSULTATS}

Chez les souches de $B$ cinerea sensibles aux dicarboximides, le fenpiclonil et l'iprodione inhibent à la fois la germination des spores et l'élongation des filaments germinatifs, ce dernier processus étant toutefois le plus sensible à ces 2 fongicides (tableau I). Les filaments germinatifs des spores traitées par le fenpiclonil, l'iprodione ou le tolclofos-méthyl apparaissent plus ramifiés et plus trapus que ceux issus de spores non traitées; parfois à des doses sublétales, ces filaments éclatent. Par ailleurs, dans ces essais sur spores comme dans ceux sur mycélium, le fenpiclonil présente une fongitoxicité environ 20 fois supérieure à celle de l'iprodione (tableau I).

Les mutants de $B$ cinerea et $P$ expansum fortement résistants à l'iprodione, le sont également vis-à-vis du fenpiclonil et du tolclofos-méthyl.

Cette résistance croisée positive est également clairement observée parmi des souches naturelles de $P$ herpotrichoides. Par contre, chez $B$ cinerea les souches issues du vignoble et présentant des niveaux de résistance compris entre 10 et 20 vis-à-vis de l'iprodione sont peu ou pas résistantes au fenpiclonil et au tolclofos-méthyl (tableaux I et II).

\section{DISCUSSION}

La similitude des effets fongitoxiques du fenpiclonil, de l'iprodione et du tolclofos-méthyl au moment de la germination des spores de $B$ cinerea, de même que la résistance croisée positive observée entre eux chez divers champignons, suggèrent que ces 3 fongicides possèdent un même mode d'action. Ce dernier reste toutefois inconnu car jusqu'à maintenant l'ensemble des travaux réalisés sur les dicarboximides (dont un représentant est l'iprodione) et les dérivés aromatiques (dont un représentant est le tolclofosméthyl) n'ont pu aboutir (Leroux et Fritz, 1984). Parmi les hypothèses avancées, celle d'une in-

Tableau I. Effet de fongicides sur diverses souches de Botrytis cinerea.

\begin{tabular}{|c|c|c|c|c|c|c|}
\hline \multirow[t]{2}{*}{ Fongicide } & \multicolumn{3}{|c|}{$\begin{array}{c}C I_{50} \text { a en mg/ldes } \\
\text { souches sensibles } B S(5)^{c}\end{array}$} & \multicolumn{3}{|c|}{$\begin{array}{l}\text { Niveaux de résistance } a, b \\
\text { des souches résistantes }\end{array}$} \\
\hline & spores & filaments & mycélium & $B r 1(8) c$ & $B r 2(2)^{c}$ & $B R(2)^{c}$ \\
\hline Fenpiclonil & $\begin{array}{c}0,1 \\
{[0,08-0,13]}\end{array}$ & $\begin{array}{c}0,02 \\
{[0,01-0,03]}\end{array}$ & $\begin{array}{c}0,008 \\
{[0,006-0,1]}\end{array}$ & $\begin{array}{c}1,1 \\
{[0,8-1,4]}\end{array}$ & $\begin{array}{c}4,5 \\
{[4-5]}\end{array}$ & $\begin{array}{c}1250 \\
{[1000-1500]}\end{array}$ \\
\hline Iprodione & $\begin{array}{c}1,5 \\
{[1-2]}\end{array}$ & $\begin{array}{c}0,4 \\
{[0,3-0,7]}\end{array}$ & $\begin{array}{c}0,2 \\
{[0,15-0,3]}\end{array}$ & $\begin{array}{c}11 \\
{[10-13]}\end{array}$ & $\begin{array}{c}16 \\
{[15-17]}\end{array}$ & $>250$ \\
\hline $\begin{array}{l}\text { Tolclofos- } \\
\text { méthyl }\end{array}$ & $>50$ & $\begin{array}{c}1,5 \\
{[1-2]}\end{array}$ & $\begin{array}{c}0,8 \\
{[0,5-1]}\end{array}$ & $\begin{array}{c}1,2 \\
{[0,6-2]}\end{array}$ & $\begin{array}{c}4,2 \\
{[4-4,5]}\end{array}$ & $>62$ \\
\hline
\end{tabular}

\footnotetext{
a : les valeurs extrêmes observées correspondent aux nombres entre crochets. ${ }^{b}$ : les niveaux de résistance sont estimés à partir des
} essais sur la croissance mycélienne; ${ }^{c}$ : nombre de souches étudiées par catégorie. 
Tableau II. Effet de fongicides sur la croissance mycélienne de diverses souches de Penicillium expansum et de Pseudocercosporella herpotrichoides.

\begin{tabular}{lccccc}
\hline Fongicide & \multicolumn{2}{c}{ P expansum } & & \multicolumn{3}{c}{$C l_{50}$ mycélium en mg/la } \\
& $P S(2)^{b}$ & $P R(3)^{b}$ & $H S(4)^{b}$ & $\begin{array}{c}\text { P herpotrichoides } \\
H r l(4)^{b}\end{array}$ & $H r 2(7)^{b}$ \\
\hline \multirow{2}{*}{ Fenpiclonil } & 0,4 & 10 & 0,4 & 2,5 & 6 \\
& {$[0,3-0,5]$} & {$[7-15]$} & {$[0,3-0,5]$} & {$[2-3]$} & {$[4-9]$} \\
Iprodione & 2,0 & $>50$ & 7 & 22 & $>30$ \\
& {$[1,5-2,5]$} & & {$[6-8]$} & {$[20-25]$} & $>30$ \\
\hline \multirow{2}{*}{ Tolclofos-méthyl } & 4,5 & $>50$ & $>30$ & $>30$ & \\
& {$[3-6]$} & & & & \\
\hline
\end{tabular}

a : les valeurs extrêmes observées correspondent aux nombres entre crochets; $b$ : nombres de souches étudiées par catégorie.

teraction de ces subtances avec des composants membranaires (enzymes ?), entraînant ensuite une peroxidation des lipides, est envisagée par Edlich et Lyr (1987). Ce mode d'action pourrait notamment être à l'origine des altérations morphologiques observées sur les filaments germinatifs chez $B$ cinerea. Quant à un effet primaire sur les mitochondries, il semble peu probable ( $R$ Fritz, communication personnelle), bien que le pyrrolnitrin soit décrit comme un inhibiteur de la chaîne respiratoire ou un découplant chez divers champignons (Lyr, 1977).

Sous l'angle de la résistance, il est intéressant de souligner la différence de comportement entre, d'une part, l'iprodione et, d'autre part, le fenpiclonil et le tolclofos-méthyl vis-à-vis des souches de $B$ cinerea moyennement résistantes aux dicarboximides, détectées dans la nature. En effet si les dicarboximides ne peuvent maîtriser ces souches en pratique, il est possible que le fenpiclonil et plus généralement les phénylpyrroles, du fait des niveaux de résistance faibles, assurent une efficacité correcte; les résultats rapportés avec le CGA 173506 semblent le confirmer (Gehmann et al, 1990). Toutefois, chez d'autres parasites, au vu des observations réalisées sur $P$ herpotrichoides, le risque de résistance en pratique ne peut être totalement exclu pour ces fongicides phénylpyrroles.

\section{RÉFÉRENCES}

Edlich W, Lyr H (1987) Mechanism of action of dicarboximide fungicides. In: Modern selective fungicides (H Lyr, ed), Longman, Londres, 107-118

Gehmann K, Nyfeler R, Leadbeater AJ, Nevill D, Sozzi D (1990) CGA 173506: a new phenylpyrrole fungicide for bread-spectrum disease control. Brighton Crop Prot Conf. Pests Dis 1990, 399-406

Leroux P. Fritz R (1984) Antifungal activity of dicarboximides and aromatic hydrocarbons and resistance to these fungicides. In: Mode of action of antifungal agents (APJ Trinci, JF Ryley, eds) Brit Mycol Soc UK, 207-237

Leroux P, Gredt M (1989) Negative cross-resistance of benzimiazole-resistant strains of Botrytis cinerea, Fusarium nivale and Pseudocercosporella herpotrichoides to various pesticides. Neth J Plant Pathol, 95 suppl 1, s121-s127

Leroux P, Gredt M, Fritz R (1978) Études en laboratoire de souches de quelques champignons phytopathogènes résistantes, à la dichlozoline, à la dicyclidine, à l'iprodione, à la vinchlozoline et à divers fongicides aromatiques. Med Fac Landbouww Rijksuniv Gent 43, 881-889

Lyr $H$ (1977) Effect of fungicides on energy production and intermediary metabolism. In: Antifungal compounds (MR Siegel, HD Sisler, eds) vol 2, M Dekker, New York, 301-332

Nevill D, Nyfeler R, Sozzi D (1988) CGA 142705: a novel fungicide for seed treatment. Brighton Crop Prot Conf Pests Dis 1988, 65-72 